\title{
Clinical and prognostic significance of signal transducer and activator of transcription 3 and mucin 1 in patients with non-small cell lung cancer following surgery
}

\author{
ZHI-GANG SUN ${ }^{1}$, MIN ZHANG $^{2}$, FEI YANG $^{3}$, WEI GAO $^{3}$, ZHOU WANG $^{4}$ and LIANG-MING ZHU ${ }^{1}$ \\ Departments of ${ }^{1}$ Thoracic Surgery, ${ }^{2}$ Dermatology and ${ }^{3}$ Pathology, Jinan Central Hospital Affiliated to \\ Shandong University, Shandong University, Jinan, Shandong 250013; ${ }^{4}$ Department of Thoracic Surgery, \\ Provincial Hospital Affiliated to Shandong University, Jinan, Shandong 250021, P.R. China
}

Received January 26, 2017; Accepted October 6, 2017

DOI: $10.3892 / \mathrm{ol} .2018 .7858$

\begin{abstract}
Signal transducer and activator of transcription 3 (STAT3) and mucin 1 (MUC1) are associated with development, progression and a poor prognosis in several types of cancer. The present study investigated the levels of STAT3 and MUC1 in patients with non-small cell lung cancer (NSCLC) following surgery. In total, 98 patients with NSCLC were enrolled into the study. STAT3, phosphorylated (p)-STAT3 and MUC1 expression in NSCLC specimens obtained from patients were investigated using immunohistochemical analysis. Enumeration results were analyzed using the $\chi^{2}$ test or Fisher's exact probability test. Spearman's rank correlation was used to analyze correlations between STAT3, p-STAT3 and MUC1 expression. Univariate analysis was conducted using the Kaplan-Meier estimator curve method and Cox regression multivariate analysis was performed in order to determine prognostic factors. Results demonstrated that STAT3 and p-STAT3 expression was identified in 82 and 51 patients, respectively. Furthermore, the expression of MUC1 was identified in 61/98 cases (62.2\%) and STAT3 expression was significantly associated with pathological tumor-node-metastasis stage (pTNM; P<0.01). p-STAT3 expression was associated with pathological type $(\mathrm{P}<0.01)$, pathological lymph nodes $(\mathrm{pN}$; $\mathrm{P}<0.01)$ and pTNM $(\mathrm{P}<0.05)$. MUC1 expression was associated with pathological type $(\mathrm{P}<0.05)$, pathological tumor $\mathrm{pT}$ $(\mathrm{P}<0.05), \mathrm{pN}(\mathrm{P}<0.01)$ and $\mathrm{pTNM}(\mathrm{P}<0.01)$. STAT3 expression was positively associated with $\mathrm{p}-\mathrm{STAT} 3$ expression $(\mathrm{P}<0.05)$ and p-STAT3 expression was positively associated with MUC1
\end{abstract}

Correspondence to: Dr Liang-Ming Zhu, Department of Thoracic Surgery, Jinan Central Hospital Affiliated to Shandong University, Shandong University, 105 JieFang Road, Jinan, Shandong 250013, P.R. China

E-mail: zlm1655@126.com

Key words: signal transducer and activator of transcription 3, phosphorylated signal transducer and activator of transcription 3, mucin 1 , non-small cell lung cancer expression $(\mathrm{P}<0.01)$. Overall, the results identified that the 3 -year survival rate was $56.1 \%$ and was significantly associated with the degree of differentiation $(\mathrm{P}<0.05)$, $\mathrm{pT}(\mathrm{P}<0.01), \mathrm{pN}$ $(\mathrm{P}<0.01)$, pTNM stage $(\mathrm{P}<0.01)$, p-STAT3 expression $(\mathrm{P}<0.01)$ and MUC1 expression $(\mathrm{P}<0.05)$. Results obtained from the Cox multivariate regression analysis demonstrated that $\mathrm{pN}$ and p-STAT3 expression were independent factors associated with the 3 -year survival rate.

\section{Introduction}

Lung cancer is the most common cause of cancer-associated mortality worldwide. Non-small cell lung cancer (NSCLC) accounts for $>85 \%$ of lung cancer cases, with squamous cell carcinoma and adenocarcinoma being the predominant subtypes (1). Patients with early-stage NSCLC typically undergo surgical therapy as it is currently the most effective form of treatment; however, $75 \%$ of patients with NSCLC present with an advanced disease stage at the time of diagnosis (2). Despite developments in diagnostic and therapeutic techniques, overall prognosis is unsatisfactory as the overall 5 -year survival rate is $\sim 15 \%$ (3). The tumor-node-metastasis (TNM) staging system (4), according to histopathologic findings, lacks sufficient predictive value as significant differences in survival are often observed for the same TNM stage. Therefore, a combination of a number of biomarkers including p53, p21, Ki-67, KRAS, and cyclin D1, to more accurately distinguish patients with NSCLC with poor survival would be valuable $(5,6)$.

Signal transduction and activators of transcription factor (STAT)3, a member of the STAT family, is frequently regarded as an oncogene (7). STAT3 can be activated via phosphorylation events within the Janus kinase-STAT and/or Ras-mitogen-activated protein kinase signaling pathways, producing tyrosine or serine phosphorylated STAT3 (p-STAT3) (8). p-STAT3 is able to increase the expression levels of certain target genes, including vascular endothelial growth factor and mucin 1 (MUC1) $(9,10)$. STAT3 may serve as one of the oncogenic critical factors in NSCLC.

MUC1 is a transmembrane glycoprotein (11). In normal tissues, it is present on the apical surface of normal glandular 
epithelial cells (12). In certain types of cancer tissue, MUC1 expression is upregulated and presented on the whole cell surface (13). MUC1 may serve an important function in tumor development and progression and is associated with a poor prognosis in certain types of cancer.

A limited number of studies have previously demonstrated the prognostic value of STAT3 and MUC1 in NSCLC. Therefore, the present study investigated the association between STAT3 expression, MUC1 expression and the clinical features of patients with NSCLC. Furthermore, the present study also investigated the potential prognostic value of using STAT3 and MUC1 expression levels to predict the survival rate of patients with NSCLC using univariate and multivariate analysis.

\section{Materials and methods}

Patients. The present study was approved by the Shandong University Ethics Committee (Shandong, China). In total, 106 consecutive patients with NSCLC who attended the Department of Thoracic Surgery, Jinan Central Hospital Affiliated to Shandong University (Shandong, China) and the Department of Thoracic Surgery East Ward, Provincial Hospital Affiliated to Shandong University (Shandong, China) between September 2011 and December 2011 were enrolled into this retrospective study. A total of 98 patients remained subsequent to use of the following inclusion criteria: i) Patients underwent a radical excision (lobectomy or pneumonectomy with regional lymph node dissection) and received confirmation of squamous cell carcinoma or adenocarcinoma via pathological analysis following surgery; ii) patients were diagnosed with stage I-IIIa NSCLC, according to the TNM staging system outlined by the International Union Against Cancer (2009); iii) patients accepted no pre-surgical radiotherapy, or chemotherapy and had no surgical contraindications; and iv) follow-up notes were well preserved. Patients who had incomplete or lost follow-up were excluded. The ages of the patients enrolled in this study ranged from 47 to 78 years (mean, 63.7 years). A total of 55 patients were men and 43 were women. A total of 52 patients had adenocarcinoma and 46 had squamous cell carcinoma. A total of 68 patients had lymph node metastasis and 30 patients did not have lymph node metastasis. A total of 24 patients had stage I disease, 52 patients had stage II disease and 22 patients had stage IIIa disease. Table I outlines patient clinicopathological characteristics.

Immunohistochemical (IHC) analysis. IHC staining for STAT3, p-STAT3 and MUC1 protein were detected using the streptavidin-peroxidase method. The experimental specimens were included in the 98 cancer tissue specimens obtained from the 98 patients with NSCLC. Adjacent non-tumorous lung tissues were used as the control tissues. The experimental specimens were fixed in $10 \%$ neutral buffered formalin (cat no. M004; Shanghai GeFan Biotechnology Company, Ltd., Shanghai, China) immediately following surgery at room temperature for no more than $24 \mathrm{~h}$. Each 4- $\mu$ m-thick section was deparaffinised, rehydrated and incubated with fresh $0.3 \% \mathrm{H}_{2} \mathrm{O}_{2}$ in methanol for $30 \mathrm{~min}$ at room temperature to block endogenous peroxidase activity. Following rehydration through a graded series of ethanol concentrations (95\% $5 \mathrm{~min}, 80 \% 5 \mathrm{~min}$, $75 \% 5 \mathrm{~min})$ at room temperature, the sections were autoclaved in $10 \mathrm{mM}$ citrate buffer $(\mathrm{pH} 6.0)$ at $120^{\circ} \mathrm{C}$ for $3 \mathrm{~min}$ and then cooled to $30^{\circ} \mathrm{C}$. Following rinsing with $0.1 \mathrm{M}$ phosphate-buffered saline (PBS; pH 7.4), nonspecific binding sites were blocked by incubation with $10 \%$ normal goat serum (cat no. WE0387-KPO; Beijing Baiao Lai Bo Technology Co., Ltd., Beijing, China, dilution, 1:100) for $30 \mathrm{~min}$ at room temperature. Sections were then incubated at $4^{\circ} \mathrm{C}$ overnight with the primary rabbit antibody against human STAT3 (cat no. CAU29097; 1:100; Spring Bioscience Corporation, Pleasanton, CA, USA), rabbit antibody against human p-STAT3 (cat no. sc-7993 tyr705; 1:100; Santa Cruz Biotechnology, Inc., Dallas, TX, USA, dilution 1:100) and rabbit antibody against human MUC1 (cat no. bs-1018R; 1:500; Bo Ao Sen Biotechnology Co., Ltd., Beijing, China) in PBS containing $1 \%$ bovine serum albumin. The sections were then washed with PBS and incubated with biotinylated peroxidase labelling sheep anti-rabbit immunoglobulin G (cat no. A100970; 1:400; Nanjing Long Kwai Biological Technology Co., Ltd., Nanjing, China) for $30 \mathrm{~min}$ at room temperature, and incubated with streptavidin-biotin peroxidase complex solution for $30 \mathrm{~min}$ at room temperature. The chromogen, 3,3'-diaminobenzidine tetrahydrochloride, was applied as a $0.02 \%$ solution containing $0.005 \% \mathrm{H}_{2} \mathrm{O}_{2}$ in $50 \mathrm{mM}$ ammonium acetate-citrate acid buffer ( $\mathrm{pH} \mathrm{6.0)}$. Finally, the sections were lightly counterstained with Mayer's haematoxylin for $30 \mathrm{~min}$ and mounted at room temperature. Specimens were visualized using the Envision System (Dako; Agilent Technologies, Inc., Santa Clara, CA, USA) and an Olympus optical microscope (magnification, x200). STAT3, p-STAT3 and MUC1 expression levels were measured using the IHC scoring system as described previously (14). Briefly, a score of 3 indicated that $>50 \%$ of the cells exhibited mild to moderate staining intensity, or $>20 \%$ of cells exhibited strong staining intensity; a score of 2 indicated that $20-50 \%$ of cells demonstrated mild to moderate staining intensity, or $20 \%$ of cells exhibited strong staining intensity; a score of 1 indicated that $<20 \%$ of cells demonstrated mild to moderate staining intensity; and a score of 0 indicated that no staining was present. A score of $\geq 2$ demonstrated positive expression.

Follow-up. In total, 74 patients received chemotherapy, 25 patients received postsurgical radiotherapy and 20 patients received epidermal growth factor receptor-tyrosine kinase inhibitor therapy. Patients were examined every 3 or 4 months during the first 3 years. During each follow-up visit the patient underwent a thorough physical examination, chest computed tomography (CT), brain CT or magnetic resonance imaging, abdomen ultrasonography or CT. In total, 6 patients underwent positron emission tomography combined with $\mathrm{CT}$ examination. The location and time of tumor relapse were recorded. Patients who succumbed to mortality due to the tumor were included in the prognostic analysis.

Statistical analysis. Enumeration data were analyzed using the $\chi^{2}$ test or Fisher's exact probability test. Spearman's rank correlation was performed to analyze correlations between 
Table I. Association between STAT3, p-STAT3 and MUC1 expression and clinical features of 98 patients with NSCLC.

\begin{tabular}{|c|c|c|c|c|c|c|c|c|c|c|}
\hline \multirow[b]{2}{*}{ Clinical features } & \multirow{2}{*}{$\begin{array}{l}\text { No. of } \\
\text { patients }\end{array}$} & \multicolumn{3}{|c|}{ STAT3 } & \multicolumn{3}{|c|}{ p-STAT3 } & \multicolumn{3}{|c|}{ MUC1 } \\
\hline & &,$- \mathrm{n}$ &,$+ \mathrm{n}$ & P-value &,$- \mathrm{n}$ &,$+ \mathrm{n}$ & P-value &,$- \mathrm{n}$ &,$+ \mathrm{n}$ & P-value \\
\hline Total & 98 & 16 & 82 & & 47 & 51 & & 37 & 61 & \\
\hline Sex & & & & $0.596^{\mathrm{a}}$ & & & 0.158 & & & 0.835 \\
\hline Male & 55 & 8 & 47 & & 30 & 25 & & 20 & 35 & \\
\hline Female & 43 & 8 & 35 & & 17 & 26 & & 17 & 26 & \\
\hline Age, years & & & & $0.176^{\mathrm{a}}$ & & & 0.842 & & & 0.682 \\
\hline$<60$ & 45 & 10 & 35 & & 21 & 24 & & 17 & 28 & \\
\hline$\geq 60$ & 53 & 6 & 47 & & 26 & 27 & & 20 & 33 & \\
\hline Tumor location & & & & $1.000^{\mathrm{a}}$ & & & 0.539 & & & 0.397 \\
\hline Central & 39 & 6 & 33 & & 17 & 22 & & 17 & 22 & \\
\hline Peripheral & 59 & 10 & 49 & & 30 & 29 & & 20 & 39 & \\
\hline Histological type & & & & $1.000^{\mathrm{a}}$ & & & 0.005 & & & 0.036 \\
\hline SCC & 52 & 9 & 43 & & 32 & 20 & & 25 & 27 & \\
\hline $\mathrm{ADC}$ & 46 & 7 & 39 & & 15 & 31 & & 12 & 34 & \\
\hline Differentiation & & & & $>0.05^{\mathrm{a}}$ & & & $>0.05^{\mathrm{a}}$ & & & $>0.05^{\mathrm{a}}$ \\
\hline Well & 15 & 1 & 14 & & 10 & 5 & & 8 & 7 & \\
\hline Moderately & 51 & 12 & 39 & & 23 & 28 & & 16 & 35 & \\
\hline Poorly & 32 & 3 & 29 & & 14 & 18 & & 13 & 19 & \\
\hline pT & & & & $>0.05^{\mathrm{a}}$ & & & $>0.05^{\mathrm{a}}$ & & & $<0.05^{\mathrm{a}}$ \\
\hline pT1 & 11 & 2 & 9 & & 6 & 5 & & 8 & 3 & \\
\hline pT2 & 73 & 14 & 59 & & 35 & 38 & & 26 & 47 & \\
\hline pT3 & 14 & 0 & 14 & & 6 & 8 & & 3 & 11 & \\
\hline $\mathrm{pN}$ & & & & $0.08^{\mathrm{a}}$ & & & $0.001^{\mathrm{a}}$ & & & $0.001^{\mathrm{a}}$ \\
\hline- & 30 & 8 & 22 & & 23 & 7 & & 19 & 11 & \\
\hline+ & 68 & 8 & 60 & & 24 & 44 & & 18 & 50 & \\
\hline pTNM & & & & $<0.01^{\mathrm{a}}$ & & & $<0.05^{\mathrm{a}}$ & & & $<0.01^{\mathrm{a}}$ \\
\hline pI & 24 & 8 & 16 & & 17 & 7 & & 17 & 7 & \\
\hline pII & 52 & 8 & 44 & & 23 & 29 & & 15 & 37 & \\
\hline pIIIa & 22 & 0 & 22 & & 7 & 15 & & 4 & 17 & \\
\hline
\end{tabular}

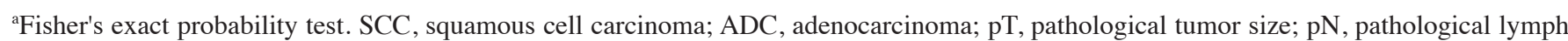
node metastasis; pTNM, pathological Tumor-Node-Metastasis.

STAT3, p-STAT3 and MUC1 expression. Univariate analysis was conducted using the Kaplan-Meier estimator curve method, and the log-rank test was used to calculate the survival rates. Cox regression multivariate analysis was performed in order to determine prognostic factors. All statistical data were analyzed using SPSS (version 13; SPSS, Inc., Chicago, IL, USA). $\mathrm{P}<0.05$ was considered to indicate a statistically significant difference.

\section{Results}

Association between STAT3, $p$-STAT3 and MUC1 expression levels and clinical characteristics. Results demonstrated that positive STAT3 expression was located within the cytoplasm and nucleus (Figs. 1 and 2), positive p-STAT3 expression was located within the nucleus (Figs. 3 and 4) and positive MUC1 expression was located within the cytoplasm (Figs. 5 and 6). STAT3 and p-STAT3 expression were identified in $83.7 \%$ (82/98) and $52.0 \%$ (51/98) of cases, respectively. MUC1 protein expression was identified in $62.2 \%$ (61/98) of cases. Table I demonstrates that increasing STAT3 expression was significantly associated with increasing pTNM stage (pI, $66.7 \%$ vs. pII, $84.6 \%$ vs. pIIIa, $100.0 \%$; $\mathrm{P}<0.01$ ), whereas p-STAT3 expression was significantly associated with pathological type (squamous cell carcinoma $38.5 \%$ vs. adenocarcinoma $67.4 \%$; $\mathrm{P}<0.01$ ), pathological lymph node (pN-23.3\% vs. pN+64.7\%.; $\mathrm{P}<0.01)$ and $\mathrm{pTNM}$ stage (pI, 29.2\% vs. pII, 55.8\% vs. pIIIa, 68.2\%; P<0.05) MUC1 expression was associated with pathological type (squamous cell carcinoma $52.0 \%$ vs. adenocarcinoma $74.0 \%$; $\mathrm{P}<0.05$ ), pathological tumor (pT1 $27.3 \%$ vs. pT2 $64.3 \%$ vs. pT3 78.6\%; $\mathrm{P}<0.05$ ), pathological lymph node ( $\mathrm{pN}-36.7 \%$ vs. $\mathrm{pN}+73.5 \%$; 


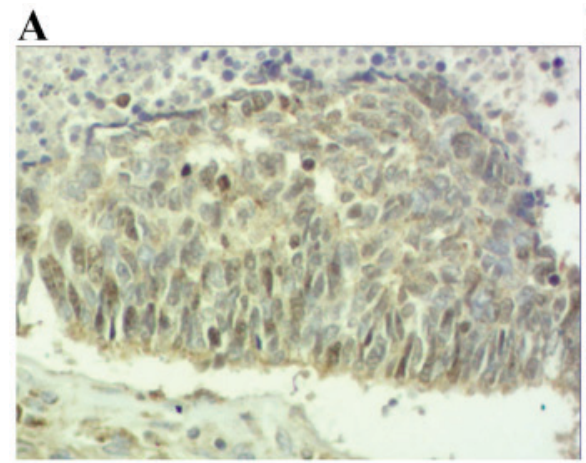

B

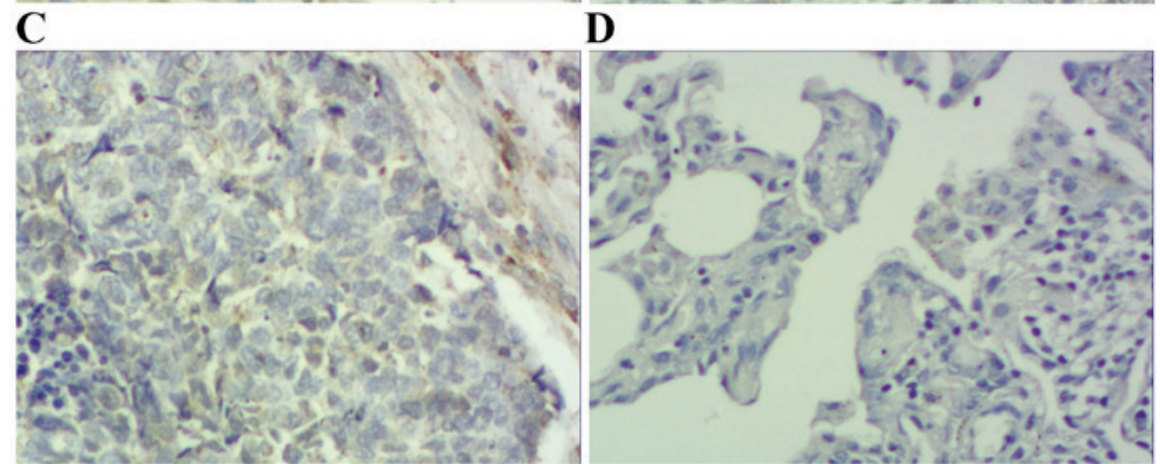

Figure 1. Immunohistochemical staining of squamous cell carcinoma tissue sections demonstrating STAT3 expression (magnification, x200). (A) Squamous cell carcinoma specimen demonstrating high expression of STAT3-positive tumor cells (score 3). (B) Squamous cell carcinoma specimen demonstrating moderate expression of STAT3-positive tumor cells (score 2). (C) Squamous cell carcinoma specimen demonstrating low expression of STAT3-positive tumor cells (score 1). (D) Photomicrographs of a corresponding normal lung tissue specimen with no STAT3-positive tumor cells (score 0) STAT3, signal transducer and activator of transcription 3 .

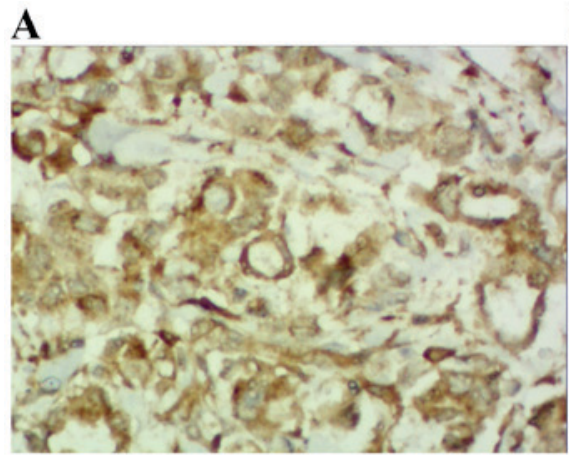

B

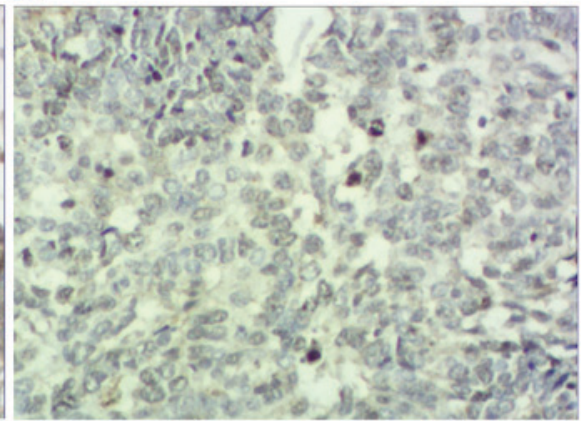

D

C

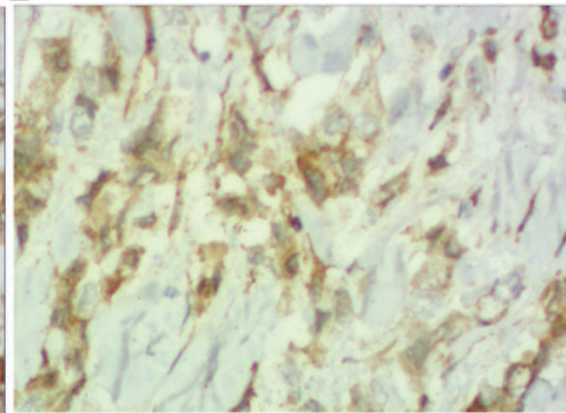

D
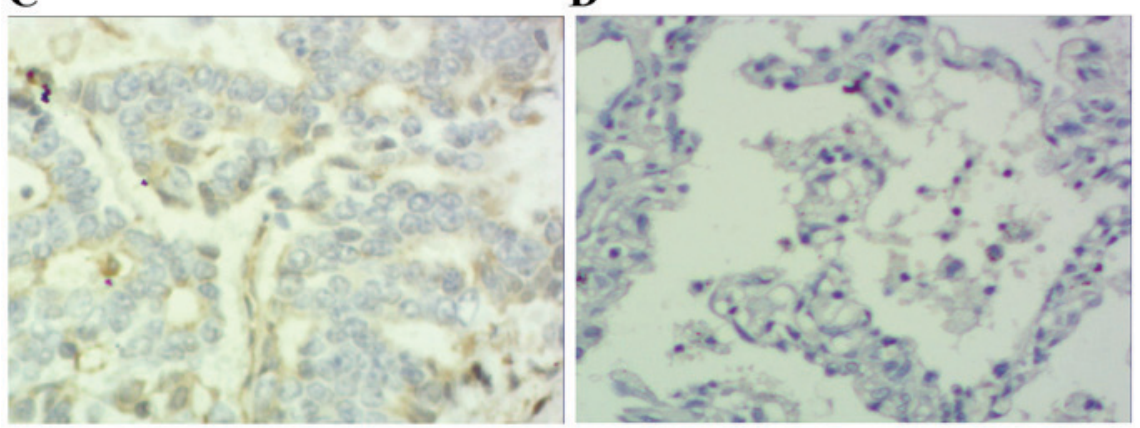

Figure 2. Immunohistochemical staining of adenocarcinoma tissue sections demonstrating STAT3 expression (magnification, x200). (A) Adenocarcinoma specimen demonstrating high expression of STAT3-positive tumor cells (score 3). (B) Adenocarcinoma specimen demonstrating moderate expression of STAT3-positive tumor cells (score 2). (C) Adenocarcinoma a specimen demonstrating low expression of STAT3-positive tumor cells (scores 1). (D) Photomicrographs of a corresponding normal lung tissue specimen with no STAT3-positive tumor cells (score 0). STAT3, signal transducer and activator of transcription 3 .

$\mathrm{P}<0.01)$ and pTNM stage (pI, $29.2 \%$ vs. pII, $71.1 \%$ vs. pIIIa, $81.8 \% ; \mathrm{P}<0.01)$. Table II demonstrates that STAT3 expression was positively correlated with $\mathrm{p}$-STAT3 expression $(\mathrm{P}<0.05)$, and that p-STAT3 expression was positively correlated with 

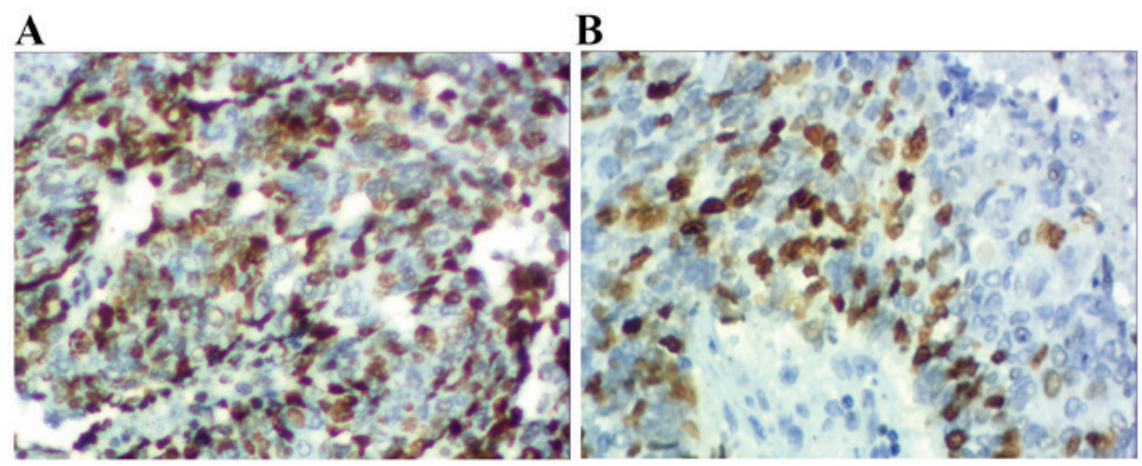

C

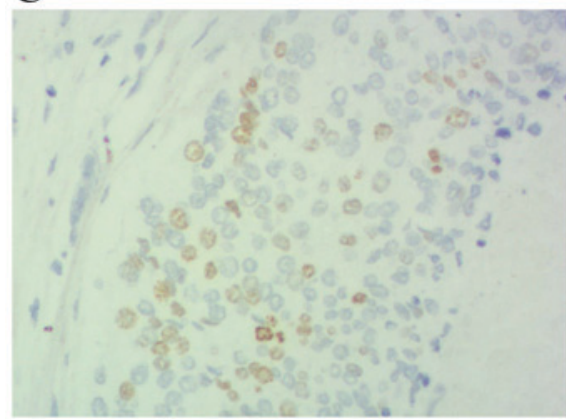

D

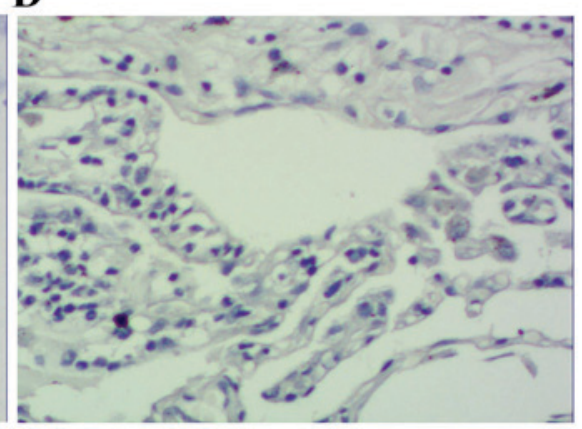

Figure 3. Immunohistochemical staining of squamous cell carcinoma tissue sections demonstrating p-STAT3 expression (magnification, x200). (A) Squamous cell carcinoma specimen demonstrating high expression of p-STAT3-positive tumor cells (score 3). (B) Squamous cell carcinoma specimen demonstrating moderate expression of p-STAT3-positive tumor cells (score 2). (C) Squamous cell carcinoma specimen demonstrating low expression of p-STAT3-positive tumor cells (score 1). (D) Photomicrographs of a corresponding normal lung tissue specimen with no p-STAT3-positive tumor cells (score 0). p-STAT3; phosphorylated signal transducer and activator of transcription 3 .

A

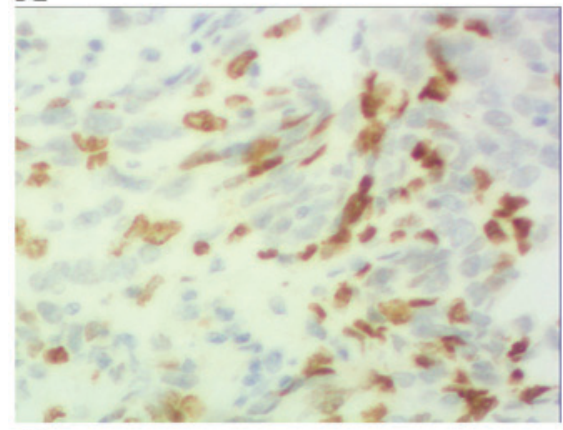

C

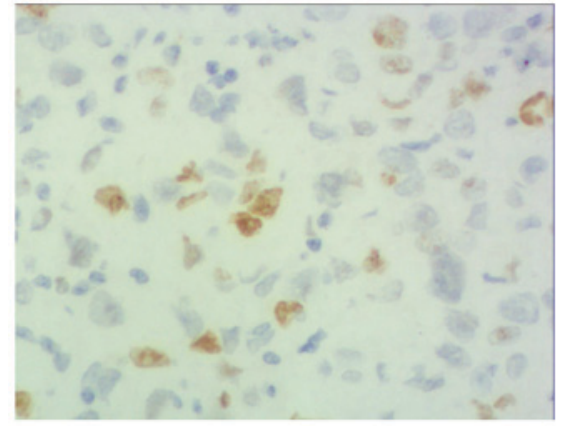

B

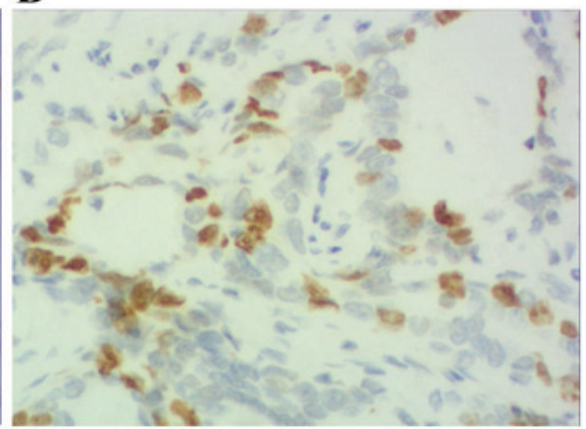

D

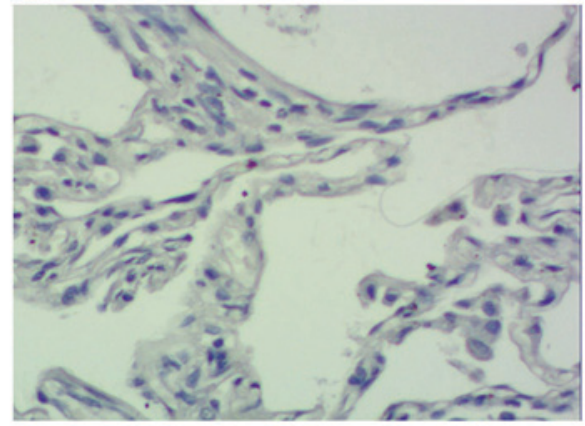

Figure 4. Immunohistochemical staining of adenocarcinoma tissue sections demonstrating p-STAT3 expression (magnification, x200). (A) Adenocarcinoma specimen with high expression of p-STAT3-positive tumor cells (score 3). (B) Adenocarcinoma specimen with moderate expression of p-STAT3-positive tumor cells (score 2). (C) Adenocarcinoma specimen with low expression of p-STAT3-positive tumor cells (score 1). (D) Photomicrographs of a corresponding normal lung tissue specimen with no p-STAT3-positive tumor cells (score 0). p-STAT3; phosphorylated signal transducer and activator of transcription 3.

MUC1 expression $(\mathrm{P}<0.01)$. There was no correlation between STAT3 and MUC1 expression $(\mathrm{P}>0.05)$.
Associations between STAT3, $p$-STAT3 and MUC1 expression levels and prognosis. The 3-year survival rate for 98 patients 


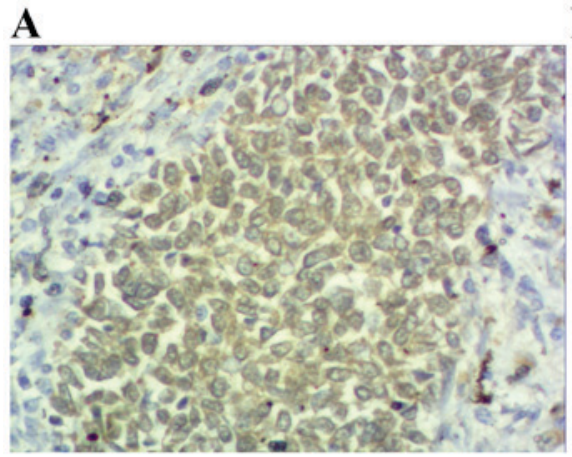

C

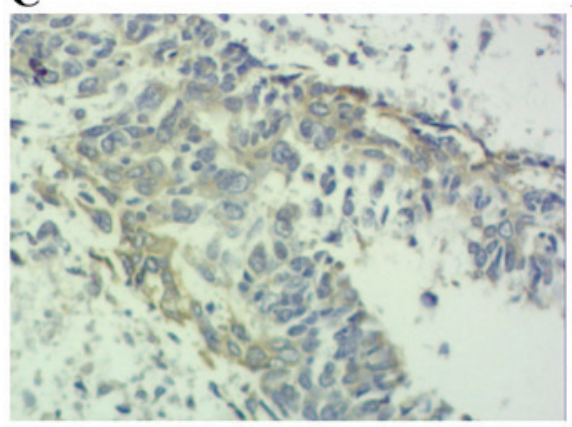

B

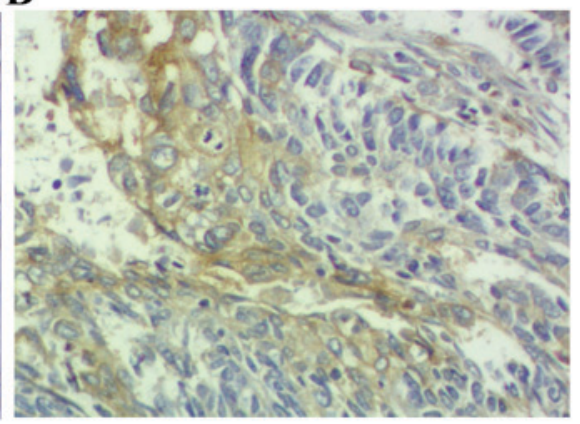

D

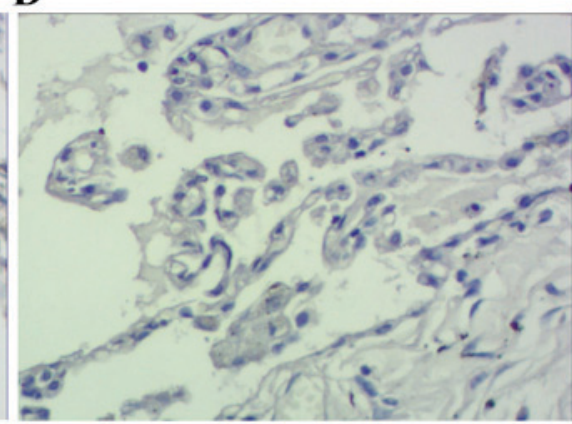

Figure 5. Immunohistochemical staining of squamous cell carcinoma tissue sections demonstrating MUC1 expression (magnification, x200). (A) Squamous cell carcinoma specimen demonstrating high expression of MUC1-positive tumor cells (score 3). (B) Squamous cell carcinoma specimen demonstrating moderate expression of MUC1-positive tumor cells (score 2). (C) Squamous cell carcinoma specimen demonstrating low expression of MUC1-positive tumor cells (score 1). (D) Photomicrographs of a corresponding normal lung tissue specimen with no MUC1-positive tumor cells (scores 0). MUC1, mucin 1.

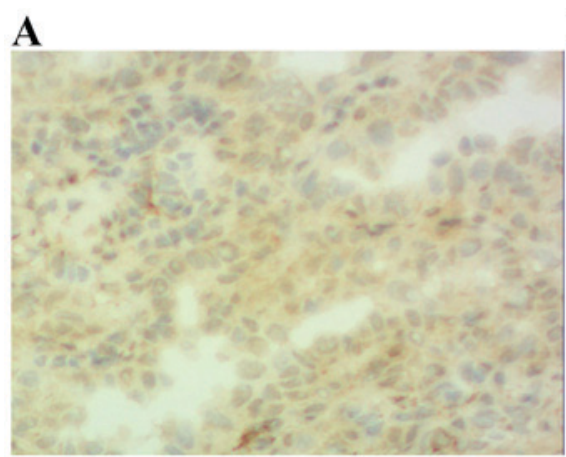

C

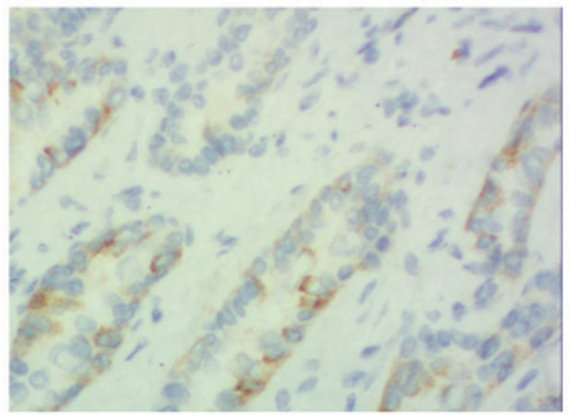

B

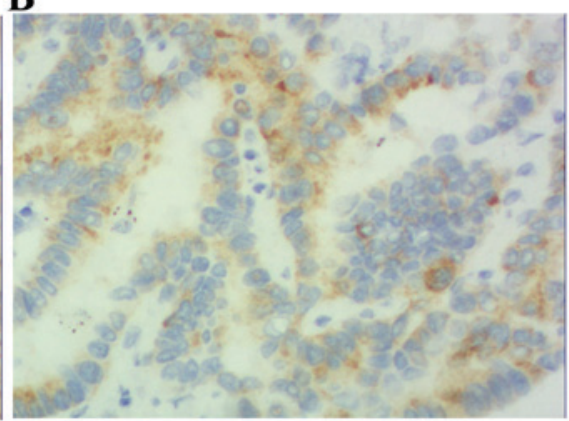

D

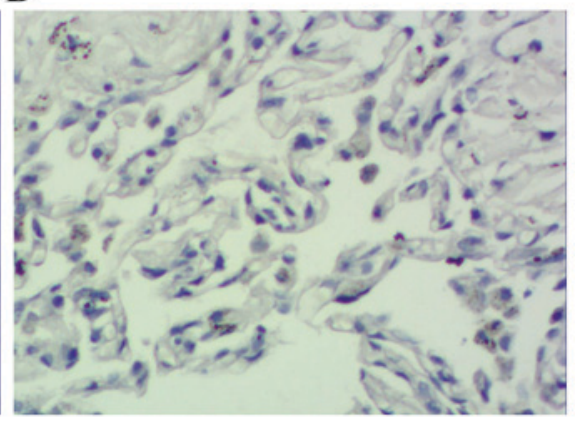

Figure 6. Immunohistochemical staining of adenocarcinoma tissue sections demonstrating MUC1 expression (magnification, x200). (A) Adenocarcinoma specimen demonstrating high expression of MUC1-positive tumor cells (score 3). (B) Adenocarcinoma specimen demonstrating moderate expression of MUC1-positive tumor cells (score 2). (C) Adenocarcinoma specimen demonstrating low expression of MUC1-positive tumor cells (score 1). (D) Photomicrographs of a corresponding normal lung tissue specimen with no MUC1-positive tumor cells (score 0). MUC1, mucin 1.

with NSCLC was $56.1 \%$. A univariate analysis was conducted using the log-rank test, and the 3-year survival rate was significantly associated with the degree of differentiation $(\mathrm{P}<0.05)$, pT $(\mathrm{P}<0.01), \mathrm{pN}(\mathrm{P}<0.01)$, pTNM stage $(\mathrm{P}<0.01)$, p-STAT3 expression $(\mathrm{P}<0.01)$ and $\mathrm{MUC1}$ expression $(\mathrm{P}<0.05)$ (Fig. 7; Table III). Cox multivariate regression demonstrated that $\mathrm{pN}$ and p-STAT3 expression were independent factors for the 3-year survival rate (Table IV). 
A

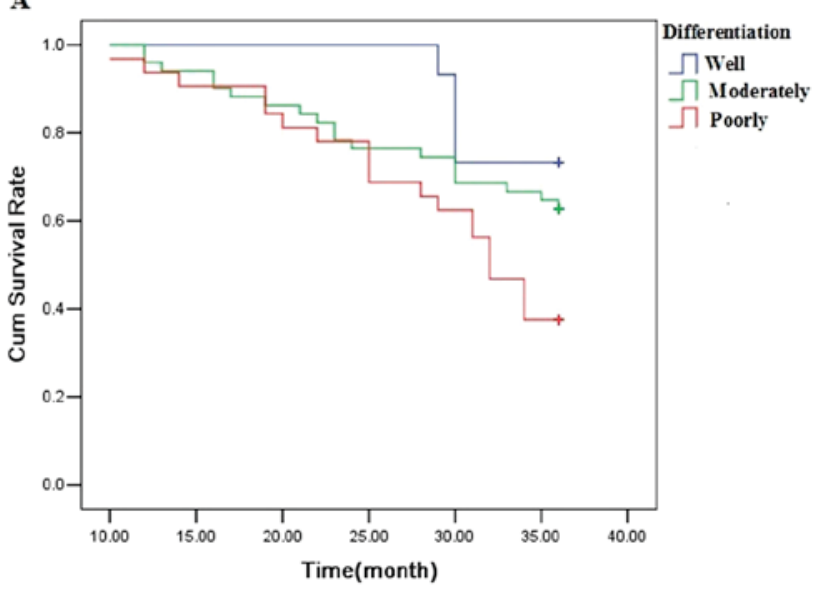

C

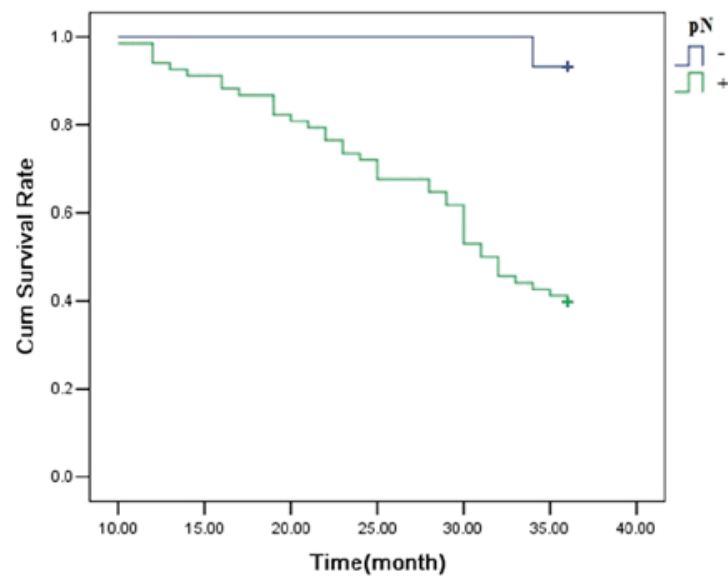

E

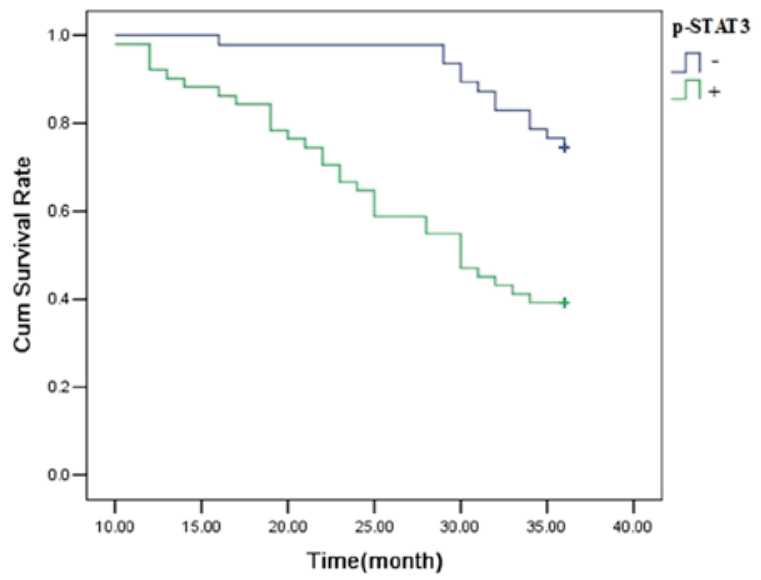

B

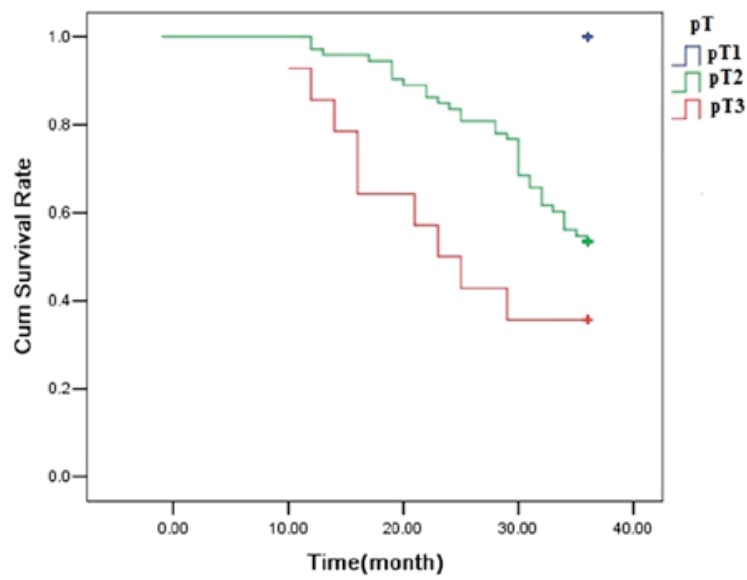

D

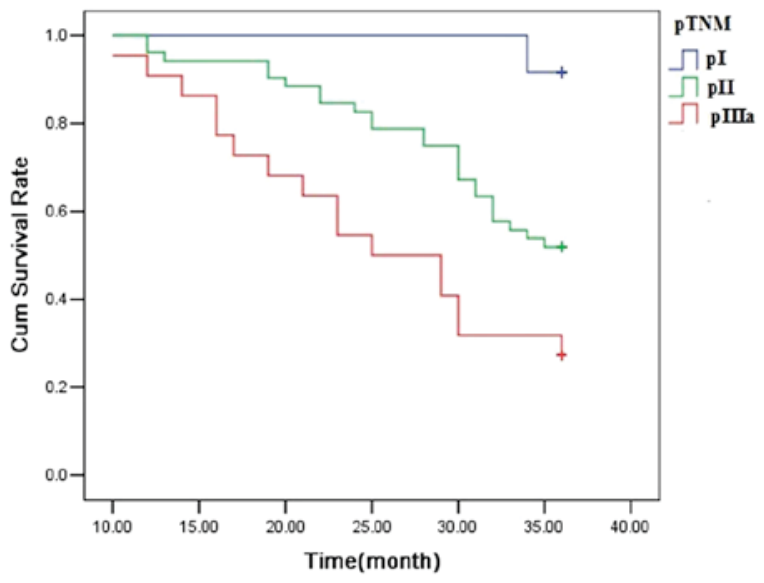

F

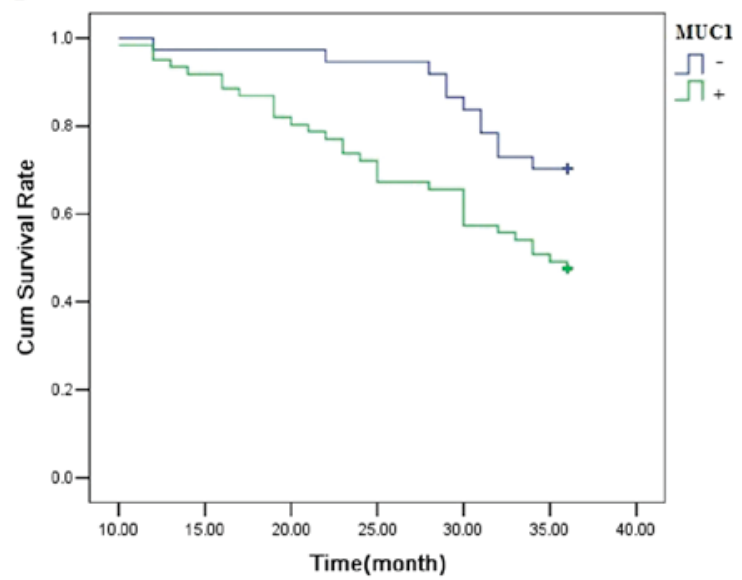

Figure 7. A Kaplan-Meier analysis of the overall survival rate after operation in patients with (A) differentiation. (B) pT. (C) pN. (D) pTNM. (E) p-STAT3expression. (F) MUC1 expression.

Table II. Association between variables in the NSCLC tissue group.

\begin{tabular}{lcc}
\hline Variable & $\mathrm{r}_{\mathrm{s}}$ & P-value $^{\mathrm{a}}$ \\
\hline STAT3 and p-STAT3 & 0.239 & 0.018 \\
STAT3 and MUC1 & 0.059 & 0.562 \\
p-STAT3 and MUC1 & 0.306 & 0.002
\end{tabular}

${ }^{\text {aS }}$ pearman's rank correlation. rs, rank correlation coefficient.

\section{Discussion}

STAT3 is regarded as a primary mediator of tumorigenesis and serves an important function in the proliferation, apoptosis and hyperplasia of tumor cells (15). Constitutively activated STAT3 has been identified in certain types of cancer, including NSCLC. In a study by Yin et al (16), a total of 76 patients with NSCLC were enrolled in the study, and the results demonstrated that STAT3 expression detected by IHC was associated with lymph node metastasis, tumor differentiation 
Table III. Univariate analysis with regard to the 3-year survival rate.

\begin{tabular}{|c|c|c|c|c|}
\hline \multirow[b]{2}{*}{ Clinical features } & \multirow[b]{2}{*}{ Patients, n } & \multicolumn{3}{|c|}{ 3-year survival rate } \\
\hline & & Patients, $n$ & Survival rate, $\%$ & P-value \\
\hline Total & 98 & 55 & 56.1 & \\
\hline Sex & & & & 0.149 \\
\hline Male & 55 & 34 & 61.9 & \\
\hline Female & 43 & 21 & 44.2 & \\
\hline Age, years & & & & 0.866 \\
\hline$<60$ & 45 & 25 & 55.6 & \\
\hline$\geq 60$ & 53 & 30 & 56.6 & \\
\hline Tumor location & & & & 0.261 \\
\hline Central & 39 & 19 & 48.7 & \\
\hline Peripheral & 59 & 36 & 61.0 & \\
\hline Histological type & & & & 0.060 \\
\hline SCC & 52 & 33 & 63.5 & \\
\hline $\mathrm{ADC}$ & 46 & 22 & 47.8 & \\
\hline Differentiation & & & & 0.033 \\
\hline Well & 15 & 11 & 72.7 & \\
\hline Moderately & 51 & 32 & 62.7 & \\
\hline Poorly & 32 & 12 & 37.5 & \\
\hline pT & & & & 0.002 \\
\hline pT1 & 11 & 11 & 100 & \\
\hline pT2 & 73 & 39 & 53.4 & \\
\hline pT3 & 14 & 5 & 35.7 & \\
\hline $\mathrm{pN}$ & & & & 0.001 \\
\hline- & 30 & 28 & 93.3 & \\
\hline+ & 68 & 27 & 39.7 & \\
\hline pTNM & & & & 0.001 \\
\hline $\mathrm{pI}$ & 24 & 22 & 91.7 & \\
\hline pII & 52 & 27 & 51.9 & \\
\hline pIIIa & 22 & 6 & 27.3 & \\
\hline Chemotherapy & & & & 0.636 \\
\hline No & 24 & 14 & 58.3 & \\
\hline Yes & 74 & 41 & 55.4 & \\
\hline Radiotherapy & & & & 0.939 \\
\hline No & 73 & 42 & 57.5 & \\
\hline Yes & 25 & 13 & 52.0 & \\
\hline EGFR-TKI therapy & & & & 0.152 \\
\hline No & 78 & 47 & 60.3 & \\
\hline Yes & 20 & 8 & 40.0 & \\
\hline STAT3 & & & & 0.278 \\
\hline- & 16 & 11 & 68.8 & \\
\hline+ & 82 & 44 & 53.7 & \\
\hline p-STAT3 & & & & 0.001 \\
\hline- & 47 & 35 & 74.5 & \\
\hline+ & 51 & 20 & 39.2 & \\
\hline MUC1 & & & & 0.019 \\
\hline- & 37 & 26 & 70.3 & \\
\hline+ & 61 & 29 & 47.5 & \\
\hline
\end{tabular}


Table IV. Results of cox regression multivariate 3-year survival analysis.

\begin{tabular}{lcccccc}
\hline Variable & $\mathrm{B}$ & $\mathrm{SE}$ & Wald & P-value & HR & $95 \%$ CI \\
\hline Sex & 0.350 & 0.651 & 0.289 & 0.591 & 1.419 & $0.396-5.078$ \\
Age, years & -0.423 & 0.404 & 1.095 & 0.295 & 0.655 & $0.297-1.446$ \\
Tumor location & 0.234 & 0.491 & 0.228 & 0.633 & 1.264 & $0.483-3.307$ \\
Histological type & 0.356 & 0.523 & 0.464 & 0.496 & 1.428 & $0.512-3.977$ \\
Differentiation & 0.420 & 0.323 & 1.692 & 0.193 & 1.522 & $0.808-2.867$ \\
pT & 0.798 & 0.483 & 2.735 & 0.098 & 2.221 & $0.863-5.721$ \\
pN & 1.996 & 0.848 & 5.544 & 0.018 & 7.362 & $1.397-38.794$ \\
pTNM & 0.360 & 0.459 & 0.617 & 0.432 & 1.434 & $0.584-3.522$ \\
Chemotherapy & -0.570 & 0.466 & 1.495 & 0.221 & 0.566 & $0.227-1.410$ \\
Radiotherapy & 0.046 & 0.402 & 0.013 & 0.910 & 1.047 & $0.476-2.301$ \\
EGFR-TKI therapy & -0.204 & 0.422 & 0.234 & 0.629 & 0.815 & $0.357-1.864$ \\
STAT3 & -0.333 & 0.573 & 0.337 & 0.562 & 0.717 & $0.233-2.206$ \\
p-STAT3 & 0.898 & 0.417 & 4.634 & 0.031 & 2.454 & $1.084-5.556$ \\
MUC1 & -0.276 & 0.423 & 0.290 & 0.514 & 0.759 & $0.331-1.738$
\end{tabular}

B, regression coefficient; SE, standard error; Wald, Wald value; HR, hazard ratio; CI, confidence interval; pT, pathological tumor; pN, pathological lymph nodes; pTNM, pathological Tumor-Node-Metastasis; EGFR-TKI, epidermal growth factor receptor-tyrosine kinase inhibitor; p-, phosphorylated; STAT3, signal transducer and activator of transcription 3; MUC1, mucin 1.

and clinical staging. Ai et al (17) used IHC to detect STAT3 expression in a total of 65 patients with NSCLC and demonstrated that increased STAT3 expression was associated with tumor differentiation. In the present study, $83.7 \%$ of tumor specimens exhibited STAT3 expression. STAT3 expression was significantly associated with pTNM, and STAT3 expression in advanced-stage patients was significantly increased compared with that in early-stage patients. These results suggest that increased STAT3 expression may be a frequent event in patients with NSCLC. A previous study demonstrated that constitutively activated STAT3 was enrolled in the janus tyrosine kinase/STAT signaling pathway of NSCLC, and results demonstrated that $22-65 \%$ of patients with NSCLC exhibited positive p-STAT3 expression (18). Wang et al (19) reported that the expression of p-STAT3 in NSCLC was significantly increased compared with that in paracancerous tissue, and it was associated with smoking and the size of the tumor. $\mathrm{Xu}$ and $\mathrm{Lu}(20)$ summarized 17 trials using meta-analysis and identified that p-STAT3 expression was associated with differentiation of NSCLC. In the present study, $52.0 \%$ of NSCLC tumor specimens demonstrated p-STAT3 expression which was associated with pathological type, $\mathrm{pN}$ and pTNM. The expression levels of p-STAT3 in the adenocarcinoma group (67.4\%) were significantly increased compared with that of the squamous cell carcinoma group $(38.5 \%$; $\mathrm{P}<0.01)$. P-STAT3 expression within the lymph node metastasis group (64.7\%) was significantly increased compared with that of the group lacking lymph node metastasis $(23.3 \%$; $\mathrm{P}<0.01)$. Furthermore, p-STAT3 expression in the advanced-stage group was increased compared with that of the early-stage group (pI, 29.2\% vs. pII, 55.8\% vs. pIIIa, 68.2\%; $\mathrm{P}<0.05$ ). The present study demonstrated that STAT3 activation increases metastasis of NSCLC. Results from the present study demonstrated that the 3-year survival rate of patients with NSCLC was $56.1 \%$, and it was significantly associated with the degree of differentiation, pT, pN, pTNM stage and p-STAT3 expression. Additionally, $\mathrm{pN}$ and p-STAT3 expression were relevant independent factors for a poor prognosis.

MUC1 expression is associated with invasion, metastasis and poor survival in certain types of cancer. Previous studies demonstrated that increased MUC1 expression was present in breast cancer $(21,22)$. In gastric cancer, increased MUC1 expression was identified in primary and metastatic cancer $(23,24)$. Furthermore, increased MUC1 expression was associated with lymph node metastasis in oral, liver and pancreatic cancer (25-27). In specific types of cancer, including renal clear cell carcinoma and thyroid cancer, it was reported that increased MUC1 expression was also associated with a shorter metastasis-free survival time $(28,29)$. Collectively, these studies demonstrate a marked association between increased MUC1 expression and cancer invasion/metastasis. Few studies have reported the clinicopathological characteristics of MUC1 in patients with NSCLC. Situ et al (30) demonstrated that in patients with NSCLC, MUC1 was more frequently expressed in adenocarcinoma compared with that in squamous cell carcinoma. Furthermore, it was also demonstrated that in patients with stage IB NSCLC, MUC1 expression was associated with being an independent prognostic factor for survival rates. Demirag et al (31) identified that increased MUC1 expression was present in lung adenosquamous cancer, and was significantly associated with disease progression. Results from the present study demonstrated that MUC1 expression occurred in $62.2 \%$ of patients with NSCLC. Patients within the adenocarcinoma group demonstrated increased MUC1 expression compared with the squamous cell carcinoma group. Furthermore, MUC1 expression was also associated with $\mathrm{pT}, \mathrm{pN}$ and $\mathrm{pTNM}$. The present study demonstrated that 
increased MUC1 expression was associated with a decreased 3-year survival rate; however, MUC1 expression was not an independent factor for survival rates.

Previous studies demonstrated that STAT3 may serve an important function in tumor progression by mediating MUC1 expression. For example, Ahmad et al (32) identified that STAT3 was able to activate MUC1 transcription through the MUC1 carboxyl-terminal receptor subunit (MUC1-C) in breast cancer cells. MUC1-C and STAT3 may serve numerous functions in cancer cell survival in an autoinductive regulatory loop. Gao et al (33) demonstrated that MUC1 overexpression in NSCLC cell lines was associated with high levels of activated STAT3. Therefore, STAT transcription factors may stimulate the MUC1 promoter at the mRNA and protein level. The present study demonstrated that p-STAT3 expression was associated with positive MUC1 expression, and that p-STAT3 and MUC1 expression were correlated with pT, pN and pTNM. Results from the present study demonstrated that p-STAT3 and MUC1 were synergistically involved in the metastasis of NSCLC.

To conclude, STAT3 expression was associated with pTNM, and the expression of p-STAT3 was associated with pathological type, $\mathrm{pN}$ and pTNM. MUC1 expression was associated with pathological type, $\mathrm{pT}, \mathrm{pN}$ and pTNM in patients with NSCLC. Furthermore, the 3-year survival rate was correlated with differentiation, $\mathrm{pT}, \mathrm{pN}$, pTNM stage, p-STAT3 and MUC1 expression, with pN and p-STAT3 serving as relevant independent factors. Additionally, p-STAT3 expression and MUC1 expression exhibited a significant positive correlation in NSCLC tissue. Collectively, the results suggest that p-STAT3 and MUC1 may serve as essential biomarkers for tumor invasion and metastasis in NSCLC.

\section{Acknowledgements}

The present study was funded by The Second Group of Jinan Science and Technology Development Program (grant no. 201602204).

\section{References}

1. Li Y, Wei S, Wang J, Hong L, Cui L and Wang C: Analysis of the factors associated with abnormal coagulation and prognosis in patients with non-small cell lung cancer. Zhongguo Fei Ai Za Zhi 17: 789-796, 2014 (In Chinese).

2. Sun W, Song L, Ai T, Zhang Y, Gao Y and Cui J: Prognostic value of MET, cyclin D1 and MET gene copy number in non-small cell lung cancer. J Biomed Res 27: 220-230, 2013.

3. Molina JR, Yang P, Cassivi SD, Schild SE and Adjei AA: Non-small cell lung cancer: Epidemiology, risk factors, treatment, and survivorship. Mayo Clin Proc 83: 584-594, 2008.

4. Mirsadraee S, Oswal D, Alizadeh Y, Caulo A and van Beek E Jr: The 7th lung cancer TNM classification and staging system: Review of the changes and implications. World J Radiol 28: 128-134, 2012.

5. Tan Z, Yang C, Zhang X, Zheng P and Shen W: Expression of glucose transporter 1 and prognosis in non-small cell lung cancer: A pooled analysis of 1665 patients. Oncotarget 8: 60954-60961, 2017.

6. Zhou J, Yu Y, Pei Y, Cao C, Ding C, Wang D, Sun L and Niu G: A potential prognostic biomarker SPC24 promotes tumorigenesis and metastasis in lung cancer. Oncotarget 8: 65469-65480, 2017.

7. Geiger JL, Grandis JR and Bauman JE: The STAT3 pathway as a therapeutic target in head and neck cancer: Barriers and innovations. Oral Oncol 56: 84-92, 2016.
8. Banerjee $\mathrm{K}$ and Resat H: Constitutive activation of STAT3 in breast cancer cells: A review. Int J Cancer 138: 2570-2578, 2016.

9. Wang H, Byfield G, Jiang Y, Smith GW, McCloskey M and Hartnett ME: VEGF-mediated STAT3 activation inhibits retinal vascularization by down-regulating local erythropoietin expression. Am J Pathol 180: 1243-1253, 2012.

10. Kondo S, Yoshizaki T, Wakisaka N, Horikawa T, Murono S, Jang KL, Joab I, Furukawa M and Pagano JS: MUC1 induced by Epstein-Barr virus latent membrane protein 1 causes dissociation of the cell-matrix interaction and cellular invasiveness via STAT signaling. J Virol 81: 1554-1562, 2007.

11. Gendler SJ, Lancaster CA, Taylor-Papadimitriou J, Duhig T, Peat N, Burchell J, Pemberton L, Lalani EN and Wilson D: Molecular cloning and expression of human tumor-associated polymorphic epithelial mucin. J Biol Chem 265: 15286-15293, 1990.

12. Jarrard JA, Linnoila RI, Lee H, Steinberg SM, Witschi H and Szabo E: MUC1 is a novel marker for the type II pneumocyte ineage during lung carcinogenesis. Cancer Res 58: 5582-5589, 1998.

13. Ho SB, Niehans GA, Lyftogt C, Yan PS, Cherwitz DL, Gum ET, Dahiya R and Kim YS: Heterogeneity of mucin gene expression in normal and neoplastic tissues. Cancer Res 53: 641-651, 1993.

14. Mizoguchi M, Betensky RA, Batchelor TT, Bernay DC, Louis DN and Nutt CL: Activation of STAT3, MAPK, and AKT in malignant astrocytic gliomas: Correlation with EGFR status, tumor grade, and survival. J Neuropathol Exp Neurol 65: $1181-1188,2006$.

15. Zhou W, Bi X, Gao G and Sun L: miRNA-133b and miRNA-135a induce apoptosis via the JAK2/STAT3 signaling pathway in human renal carcinoma cells. Biomed Pharmacother 84: 722-729, 2016.

16. Yin Z, Zhang Y, Li Y, Lv T, Liu J and Wang X: Prognostic significance of STAT3 expression and its correlation with chemoresistance of non-small cell lung cancer cells. Acta Histochem 114: 151-158, 2012.

17. Ai T, Wang Z, Zhang M, Zhang L, Wang N, Li W and Song L: Expression and prognostic relevance of STAT3 and cyclin D1 in non-small cell lung cancer. Int J Biol Markers 27: e132-e138, 2012.

18. Yu Y, Zhao Q, Wang Z and Liu XY: Activated STAT3 correlates with prognosis of non-small cell lung cancer and indicates new anticancer strategies. Cancer Chemother Pharmacol 75: 917-922, 2015.

19. Wang RJ, Zhang JZ and Wang P: Expression of pSTAT3 in non-small cell lung cancer and its clinical significance. Xi Bao Yu Fen Zi Mian Yi Xue Za Zhi 28: 288-290, 2012 (In Chinese).

20. Xu YH and Lu S: A meta-analysis of STAT3 and phospho-STAT3 expression and survival of patients with non-small-cell lung cancer. Eur J Surg Oncol 40: 311-317, 2014.

21. Lavrsen K, Madsen CB, Rasch MG, Woetmann A, Ødum N, Mandel U, Clausen H, Pedersen AE and Wandall HH: Aberrantly glycosylated MUC1 is expressed on the surface of breast cancer cells and a target for antibody-dependent cell-mediated cytotoxicity. Glycoconj J 30: 227-236, 2013.

22. Yang E, Hu XF and Xing PX: Advances of MUC1 as a target for breast cancer immunotherapy. Histol Histopathol 22: 905-922, 2007.

23. Yonezawa S, Kitajima S, Higashi M, Osako M, Horinouchi M, Yokoyama S, Kitamoto S, Yamada N, Tamura Y, Shimizu T, et al: A novel anti-MUC1 antibody against the MUC1 cytoplasmic tail domain: Use in sensitive identification of poorly differentiated cells in adenocarcinoma of the stomach. Gastric Cancer 15: 370-381, 2012.

24. Wang XT, Kong FB, Mai W, Li L and Pang LM: MUC1 immunohistochemical expression as a prognostic factor in gastric cancer: Meta-analysis. Dis Markers 2016: 9421571, 2016.

25. Zhang K, Tang W, Qu X, Guo Q, Inagaki Y, Seyama Y, Abe H, Gai R, Kokudo N, Sugawara Y, et al: KL-6 mucin in metastatic liver cancer tissues from primary colorectal carcinoma. Hepatogastroenterology 56: 960-963, 2009.

26. Mizumoto M, Honjo G, Kobashi Y, Awane M and Matsusue S: Molecular profile of apomucin and p53 protein as predictors of malignancy in intraductal papillary mucinous neoplasms of the pancreas. Hepatogastroenterology 58: 1791-1795, 2011.

27. Hamada T, Nomura M, Kamikawa Y, Yamada N, Batra SK, Yonezawa S and Sugihara K: DF3 epitope expression on MUC1 mucin is associated with tumor aggressiveness, subsequent lymph node metastasis, and poor prognosis in patients with oral squamous cell carcinoma. Cancer 118: 5251-5264, 2012. 
28. Leroy X, Zerimech F, Zini L, Copin MC, Buisine MP, Gosselin B, Aubert JP and Porchet N: MUC1 expression is correlated with nuclear grade and tumor progression in $\mathrm{pT} 1$ renal clear cell carcinoma. Am J Clin Pathol 118: 47-51, 2002.

29. Kaira K, Murakami H, Serizawa M, Koh Y, Abe M, Ohde Y, Takahashi T, Kondo H, Nakajima T and Yamamoto N: MUC expression in thymic epithelial tumors: MUC1 may be useful marker as differential diagnosis between type B3 thymoma and thymic carcinoma. Virchows Arch 458: 615-620, 2011.

30. Situ D, Wang J, Ma Y, Zhu Z, Hu Y, Long H and Rong T: Expression and prognostic relevance of MUC1 in stage IB non-small cell lung cancer. Med Oncol 28 (Suppl 1): S596-S604, 2011.

31. Demirag F, Cakir E, Bayiz H and Eren Yazici U: MUC1 and bcl-2 expression in preinvasive lesions and adenosquamous carcinoma of the lung. Acta Chir Belg 113: 19-24, 2013.
32. Ahmad R, Rajabi H, Kosugi M, Joshi MD, Alam M, Vasir B, Kawano T, Kharbanda S and Kufe D: MUC1-C oncoprotein promotes STAT3 activation in an autoinductive regulatory loop. Sci Signal 4: ra9, 2011

33. Gao J, McConnell MJ, Yu B, Li J, Balko JM, Black EP, Johnson JO, Lloyd MC, Altiok S and Haura EB: MUC1 is a downstream target of STAT3 and regulates lung cancer cell survival and invasion. Int J Oncol 35: 337-345, 2009.

(c) (i) (-) $९$ This work is licensed under a Creative Commons Attribution-NonCommercial-NoDerivatives 4.0 International (CC BY-NC-ND 4.0) License. 